\title{
Multimodal Volume-Based Tumor Neurosurgery Planning in the Virtual Workbench
}

\author{
Luis Serra ${ }^{1}$, Ralf A. Kockro ${ }^{1,2}$, Chua Gim Guan ${ }^{1}, \mathrm{Ng} \mathrm{Hern}^{1}$ \\ Eugene C.K. Lee ${ }^{1}$, Yen H. Lee ${ }^{1}$, Chumpon Chan ${ }^{2}$ and Wieslaw L. Nowinski ${ }^{1}$ \\ ${ }^{1}$ Kent Ridge Digital Labs (formerly, the Institute of Systems Science, ISS), \\ 21 Heng Mui Keng Terrace, Singapore 119613 \\ \{luis, ralf, ggchua, nghern, cklee, yhlee, wieslaw\}@krdl.org.sg \\ ${ }^{2}$ Dept. of Neurosurgery, Singapore General Hospital, Singapore
}

\begin{abstract}
We present a multimodal volume-based pre-operative surgical planning system developed around the KRDL Virtual Workbench, a mirror-based Virtual Reality system for precise work with 3D data. The planning system is being developed in collaboration with the Singapore General Hospital (SGH), and is undergoing clinical evaluation at SGH and Tan Tock Seng Hospital where 16 cases have been planned. We give a systems perspective on the user interface, introduce the multimodal aspects of the volume rendering technique, and describe the stages involved in creating the final multimodal image for planning with a clinical case: registration, segmentation of tumors, bone removal, path planning and measurements.
\end{abstract}

Keywords. Virtual Reality, neurosurgery planning, multimodal volume rendering

\section{Goal: Patient-Specific Tumour Neurosurgery Planning}

Effective planning of neurosurgery requires interaction with a complete and accurate picture of the patient's 3D pathology and surrounding anatomy. This involves two kinds of technologies: one for presenting the anatomy in many different, combined ways and seeing it from many viewpoints, and another for interacting with it. Multimodal volumetric rendering in real-time is thus our visualization of choice. Volume rendering works directly from the available scan images, and delivers a good degree of fidelity to the original images (unlike surface rendering). This is essential to show the interrelations of the different tissues for a same patient. Real-time rendering is obviously desirable to allow quick exploration of the volume, inviting the surgeon to explore viewpoints.

It is also important in planning to be able to perform operations that are $3 \mathrm{D}$ in nature, such as segmenting the tumor, measuring distances, areas and volumes, specifying cross-sections at non-orthogonal angles and planning of the path of approach. This combination of accuracy (in reporting detail from the original) and the need for a way to interact in three dimensions demands a system that can integrate volumetric rendering and 3D interaction.

We have been applying a Virtual Reality (VR) interface to the field of neurosurgery in collaboration with the department of neurosurgery Singapore General Hospital. The interface is called The KRDL Virtual Workbench [7][11], a mirror-based system: the user sits comfortably wearing stereoscopic glasses and enjoys precise hand-eye coor- 
dination by 'reaching in' into a 3D virtual image behind the mirror, with both hands. In this paper, we describe VIVIAN (Virtual Intracranial Visualization and Navigation), a tumor (frameless) neurosurgery patient-specific planning system built around the Virtual Workbench. Our approach to planning is more efficient, faster and natural than conventional methods clinically available.

\section{Background}

Neurosurgery is inherently a 3D activity, where surgical instruments have to be reliably guided to targets with millimetric accuracy. Although computer generated 3D views are common in clinical planning, precise $3 \mathrm{D}$ interaction with them is still rare. This is because the manipulative capabilities of common input devices such as the 2D mouse and keyboards do not match the volumetric manipulation. Moreover, the possibilities of $3 \mathrm{D}$ interaction go beyond what is practical using the mouse.

Great progress has been made in planning neurosurgery (see for example [6]). There are several commercially available packages that support neurosurgical patient-specific planning (pre- and intra-operative) using 3D views: e.g, Elekta's SurgiPlan, Radionics' Stereoplan, BrainLAB's BrainMAP 3D, Philips' EasyVision CT/MR and VolumeView $C T / M R$, Leibinger's STP [12]. All these systems display 3D views of the preoperative data co-registered with the patient and stereotactic equipment. However, user interaction with the planning software is achieved with a conventional mousescreen setup. Three-dimensional interaction with the preoperative data takes place during surgery, where the software guides the neurosurgeon, who holds a 3D pointer (tracked by video cameras, infrared, or other methods) that is displayed registered with the preoperative data. These interaction, although 3D, is usually limited due to the constrains of surgery to pointing at structures of interest (with the pointer or with the focus of the microscope) and little manipulation of the data and models is available to the neurosurgeon.

Interaction in $3 \mathrm{D}$ is not only more natural but provides a wider spectrum of operations. Three dimensional interfaces have been proposed for the planning: for example, Goble et al. 1995 [4] describe a 3D interface for image exploration, using hand-held props. The props approach is intuitive but the 3D interactions rely entirely on relative visual parallax and other non-stereoscopic depth cues, which tends to be inefficient due to unnecessary to and fro movement when approaching an object. Systems like Boston Dynamics [1] or SRI International [5], although using a similar setup to ours (with the addition of force feedback), concentrate in training dexterity skills and not patient-specific planning.

In the Brain Bench [8] we used the Virtual Workbench to integrate an electronic atlas of brain structure with a virtual stereotactic frame in a system which allowed faster specification of target points, improved avoidance of sensitive structures and speedier, more effective training. The Brain Bench had limited visualization capabilities (only triplanar support) and concentrated on planning the path of the virtual probe of the stereotactic frame relative to the brain atlas structures. 


\section{VIVIAN - An Overview}

VIVIAN combines a VR user interface with a volume rendering graphics engine to provide an environment for neurosurgical planning (see [3] for a short description). In VIVIAN, one of the user's hands controls the patient's volume data, turning and placing it as one would do with an object held in real space. The tool in the other hand appears as an 'stylus' or virtual tool, and performs detailed manipulation, using 3D widgets (interaction gadgets) to interact with the surgical planning tools (refer to Fig. 1 during this discussion). In all interactions, the approach is 'reach in for the object of interest and press the switch on the stylus to interact with it'. When the tip of the virtual tool enters the volume of influence around an object, the object signals that it is reactive by a colour change or other highlighting. When the tool enters a widget's volume of influence, the tool becomes the manipulator associated with that widget, and knows how to interact with it.

A 'toolrack' menu at the bottom switches between stylus modes. The buttons display their function, which may then be enabled by clicking. A slider scales the whole head ensemble, allowing detailed or overall inspection and manipulation.

Since VIVIAN deals with a variable number of objects of different and overlapping sizes (volumetric as well as polygonal), efficient selection of the working object with the stylus is important. A pop-up menu appears when clicking is done outside all objects, and remains visible while the digital switch is pressed. While the pop-up menu is up, the user can directly select an object. The list of available objects is overlaid on the display at the top-left corner. This provides feedback on the status of the objects: visible or hidden, 'selected' (indicated by a small arrow on the left), object closest to the tip of stylus (indicated by a highlight of the name) and order of alignment for registration. When objects occupy the same space (a common occurrence) highest priority is given to the object which is currently selected, followed by the position in the object list. .

\subsection{Volume Rendering with 3D Textures}

We achieve real-time volume rendering by means of 3D textures [2]. This approach achieves real-time performance by relying on the Silicon Graphics hardware-accelerated 3D texture mapping. Briefly, the algorithm takes a volume and generates sample planes orthogonal to the viewing direction, in back to front order, mapping a texture to each sample plane and blending it with the rest. The texture is obtained from the intersection between the sample plane and the volume, by trilinear interpolation. Our implementation enables free movement of the volume in 3D space and speeds up the display by having a number of sample planes that decreases with the distance to the viewpoint [9].

Multimodal support is necessary to simultaneously display complementary images in the same virtual space. Volume rendering with $3 \mathrm{D}$ textures has an inherent problem with this, since the shape of the volume is not accounted for in the depth buffer, which receives only polygons (the sampled planes). When a second set of sampled planes is rendered over another, the depth buffer disables the write over the first volume's planes, which results in an incorrect image (see Fig. 2a: MRI and MRA extents over- 
lap, but since MRA is rendered first, MRI is excluded within the MRA's extent). Even if the depth buffer is disabled, the composition would still be incorrect, since the blending function used to composite the image is order dependent (back to front).

To avoid this depth buffer problem, the traditional solution is to interleave the sample planes of the overlapping volumes, sorting them by depth value. This method composites the volumes producing a correct picture at the expense of texture binding time. Binding, the process of getting the volume texture ready for operation, is time consuming if the texture has to be reloaded, and therefore should be avoided when possible. This reloading is very likely to happen in the interleaving method given that, although a volume by itself might fit into texture memory, the combination of two (or more) is less likely to do so. The main drawback of the interleaving approach is that it requires one bind per sample plane per volume.

Another method is to composite the volumes at the image level (combining slices of the different volumes into a single set of slices). This method is not applicable if the positions of the volumes have to be controlled independently (registered together, for example). Also, it is hard to find the extent of overlap of the texels, specially from volumes that have different orientations.

Our solution capitalizes on the 'multisampling mask' option available in SGI workstations which then enables to render the volumes one at a time. When multisampling is enabled, each pixel is allocated $n$ 'samples', in effect dividing it into $n$ subpixels. Depending on the transparency value of each voxel (ranging from 0.0 to 1.0), the mask is modified so that [transparency* $n$ ] of the subpixels is written. This method is efficient in terms of texture bindings (one volume is bound at a time), is easy to implement, and most importantly for surgical planning, gives a correct visual position of the volumes. Since this method combines volumes based on a limited number of samples per pixel with a mask, one objection would be how faithful the resulting image is (compared to what would be obtained using traditional volume rendering). Another shortcoming is that the approach is volume-order dependent (different order in the rendering can produce different images). These two problems are not significant for our purposes. Firstly, the images produced are almost identical: compare Fig. $2 \mathrm{c}$ and d, which use four samples per pixel, the maximum available to us - better images can be obtained if more samples per pixel are available. Fig. $2 c$ renders MRI first, and (d) MRA first. Second, since our main objective is to determine positional relationships, fidelity to the traditional volume rendering is not the issue, as long as positional accuracy is preserved. Fig. $2 b$ shows an image obtained by combining the same two volumes but having the MRA displayed without the mask enabled: the resulting image shows more detail. But if we reverse the order of rendering, this produces the wrong picture (Fig. 2a is an example of that).

3.1.1 Volume Exploration Tools. Planning involves patient data (volumetric) exploration. A roaming tool was added to the standard volume tools (cut-box tool to provide mixed orthogonal and volumetric views by removing a wedge of the volume, or a clip tool to position and orient of six arbitrary-orientation clipping planes). This tool allows to cut and roam inside several multimodal data sets cropping them simultaneously. Real-time performance is maintained by displaying only a part of the total volume 
while keeping all objects in view. In this way, a cross section of all objects can be studies to reveal where and how tumors and vessels intersect (see Fig. 3a).

\subsection{Registration}

VIVIAN provides a set of tools for performing direct transformations on an object, such as translation, scaling and rotation. Additionally, an automatic n-point alignment tool to register more difficult cases is available. This requires at least four corresponding landmarks from source and destination objects, and uses the least-square approximation method. The more landmarks specified, the better the fit.

\subsection{Segmentation: Highlighting Pathology}

VIVIAN features a user-controlled crop-and-clone facility to segment out volumetric objects from the MRI volume. Each volume has its own Colour Look-Up Table (CLUT), a 3D widget that interactively maps scan values to external appearance. The transparency control of this widget is an effective segmentation tool to make invisible specific regions and isolate regions of interest. (This is possible with tumors that have significant density contrast against the surrounding tissue). Fig. 4 illustrates how to segment a tumor by increasing the transparency (lower white control line) of the surrounding tissue. Fig. 1 shows CT, MRA and a tumor all segmented this way.

3.3.1 Outlining Tumors. When there is no significant density contrast between a tumor and the surrounding tissue, an outlining tool is available to let the surgeon decide the extent of the tumor. The outlining tool lets the surgeons draw outlines over the original scan slices (see Fig. 5). Although the drawing is done on a slice-by-slice basis, the volume of interest remains in context during the outlining, facilitating the appreciation of the shape of the tumor.

Once the outline is completed, the surgeon can verify its extent against the volume, and correct it. Additionally, the outline can be displayed as a surface, and can be coloured and rendered with different degrees of transparency to study its relationship to the rest of the anatomy. The surgeon can then go back to the outlining tool and adjust its contours to achieve a better fit.

\subsection{Planning: Interacting with the Data}

3.4.1 Voxel Editing. The ability to interactively change the properties of individual voxels or groups of voxels is useful in planning. This allows turning voxels transparent, to see through them (thus simulating a craniotomy or a suction tool), or painting them in a different colour (for marking purposes), or restoring their original values (to correct interaction errors): Fig. 3 b.

The surgeon can select the shape of the voxel tool (rectangular, cylindrical and spherical) and its size (pointing upwards and clicking to make it bigger, or downwards to make it smaller). Without pressing the switch, the voxel tool works in 'inspect' mode: the voxels covered by the tool's shape are not shown, revealing the tissue behind. When the switch is pressed, the tool removes the voxels.

3.4.2 Measurement and Trajectory Planning. It is important in surgical planning to measure distances between points in 3D space and to draw lines that guide surgical 
approaches. VIVIAN uses markers (adjustable line segments that display length) for both purposes. The marker end-points can be moved by reaching into them with the stylus, and are either free-moving, or snapped to the nearest orthogonal axis. This is a simple and yet essential feature that capitalizes on the ease and directness of reach on the Virtual Workbench. Fig. 1 shows several trajectory paths planned on the lower-left side of the head.

\section{Case Example}

Our planning system has been used by neurosurgeons from the Singapore General Hospital and the Tan Tock Seng Hospital to plan neurosurgical approaches in 16 cases. We illustrate the planning procedure with a clinical case of a 65 year old male with a skull base tumor (meningeoma) in the petroclival region.

Three days before surgery, the patient was scanned with a contrast enhanced MRI (gradient echo) and a MRA (time of flight) both extending over the same field of view (FOV). The patient's head was fixed firmly during the scanning procedure to ensure a good registration. To visualize the bone, the skull base was scanned with a CT of $1 \mathrm{~mm}$ slice thickness. During both MR and CT procedures the patient had fiducial markers attached to his skin.

Once the data was available in VIVIAN, fusion of MRI and MRA was achieved easily since the orientation and the FOV were the same. The position and extent of coverage in the $z$-axis differed and were adjusted manually (moving exclusively along $z$; MRA displayed as volumetric vessels, MRI made semi-transparent, or as triplanar structure). Subsequently MRI/MRA and CT were fused by manually placing landmarks on the fiducials.

The tumor was segmented easily using the CLUT method, since the meningeoma showed uniform contrast-enhancement (Fig. 4).

Two possible approaches to the lesion were outlined and measured. Using the voxel editing tool, a suboccipital and a temporal craniotomy were simulated and parts of the tumor were removed respectively (Fig. 3b). It was concluded that complete removal could probably only be achieved by approaching the lesion along those two trajectories and this assumption was intra-operatively confirmed to be correct.

\section{Implementation}

VIVIAN is written with the KRDL BrixMed C++/OpenGL software toolkit [10] and runs on a Silicon Graphics Onyx2 (1 R10000 $180 \mathrm{Mhz}$ CPU, 1 Raster Manager with 64MB texture memory). The input devices used are the FASTRAK from Polhemus, with two receivers: one stylus, and one normal receiver with a digital hand switch attached. For multimodal volumes of less than $64 \mathrm{MB}$ (the available hardware texture) that occupy a quarter of the total screen space (1024x768 pixels) we obtained an average performance of 10 frames per second, in stereo. When the volumes occupy more than half of the screen space, the speed falls to 5 frames per second, due to overloading of the single Raster Manager. 


\section{Conclusions and Further Work}

Neurosurgeons value the efficient and comprehensive way in which the system allows them to understand the complexity of anatomical and pathological relationships surrounding a lesion. They all agreed that the preoperative experience of planning the approach virtually remains in the neurosurgeon's mind and is supportive during the operative procedure.

It takes less than two hours to prepare the images for the neurosurgeon (such as the ones presented in this paper), from the moment the images are available from the scanners until the neurosurgeon can start the planning. This includes off-line image conversion, registration, CLUT control, and tumor segmentation. We intend to carry the planning into the operating theatre by modifying the interface to suit a microscope.

Neurosurgeons with no prior experience with the Virtual Workbench can reach a sufficient level of familiarity with the system in approximately 20 minutes. The interface is undergoing simplification, but still maintaining the tools necessary to plan a complex procedure.

Acknowledgments. We would like to acknowledge the contribution to the project of Dr. Yeo Tseng Tsai (Dept. Neurosurgery) and Dr. Robert Tien and Dr. Sitoh Yih Yian (Dept. Radiology) of the Tan Tock Seng Hospital, and also of Dr. Tushar M. Goradia, Dept. Neurosurgery, Johns Hopkins Hospital.

\section{References}

1. Boston Dynamics, Inc. 614 Massachusetts Avenue, Cambridge, MA 02139, USA

2. Cabral, B., Cam, B., Foran, J.: Accelerated Volume Rendering and Tomographic Reconstruction Using Texture Mapping Hardware, Proc. ACM/IEEE 1994 Symposium Volume Visualization., (1994) 91-98 and 131.

3. Chua, G.G., Serra, L., Kockro, R.A., Ng, H., Nowinski, W.L., Chan, C., Pillay, P.K.: Volumebased tumor neurosurgery planning in the Virtual Workbench, Proc. IEEE VRAIS'98, (1998) 167-173.

4. Goble, J. C. Hinkley, K., Pausch, R., Snell, J. W. and Kassell, N. F.: Two-handed spatial interface tools for neurosurgical planning, IEEE Computer, 28 (7), (1995) 20-26.

5. Hill, J.W., Holst, P.A., Jensen, J.F., Goldman, J., Gorfu, Y., Ploeger, D.W., Telepresence Interface with Applications in Microsurgery and Surgical Simulations, Proc. MMVR:6, (1997) 96-102.

6. Kikinis, R., Gleason, P.L., Moriarty, T.M., Moore, M.R., Alexander, E., Stieg, P.E., Matsumae, M., Lorensen, W.E., Cline, H.E., Black, P., and Jolesz, F.A.: Computer Assisted Interactive Three-Dimensional Planning for Neurosurgical Procedures. Neurosurgery, 38 (4) (1996) 640-651.

7. Poston, T. and Serra, L.: Dextrous Virtual Work, Communications of the ACM, 29 (5), (1996) $37-45$.

8. Serra, L., Nowinski, W.L., Poston, T., Chua, B.C., Ng H., Lee, C.M., Chua, G.G., Pillay, P.K.: The Brain Bench: Virtual Tools for Neurosurgery, J. Medical Image Analysis, 1 (4), (1997) 317-329.

9. Serra, L., Ng, H., Chua, B.C. and Poston, T.: Interactive vessel tracing in volume data, Proc. ACM Symposium of Interactive 3D Graphics, (1997) 131-137.

10. Serra, L. and Ng, H.: The BrixMed C++ Applications Programming Interface, ISS Internal Technical Report. (1997)

11. The Virtual Workbench Web Page: http://www.krdl.org.sg/RND/biomed/virtual/index.html

12. The 65th Annual Meeting of the American Association of Neurological Surgeons, Scientific Program, (1997) 71-81. 


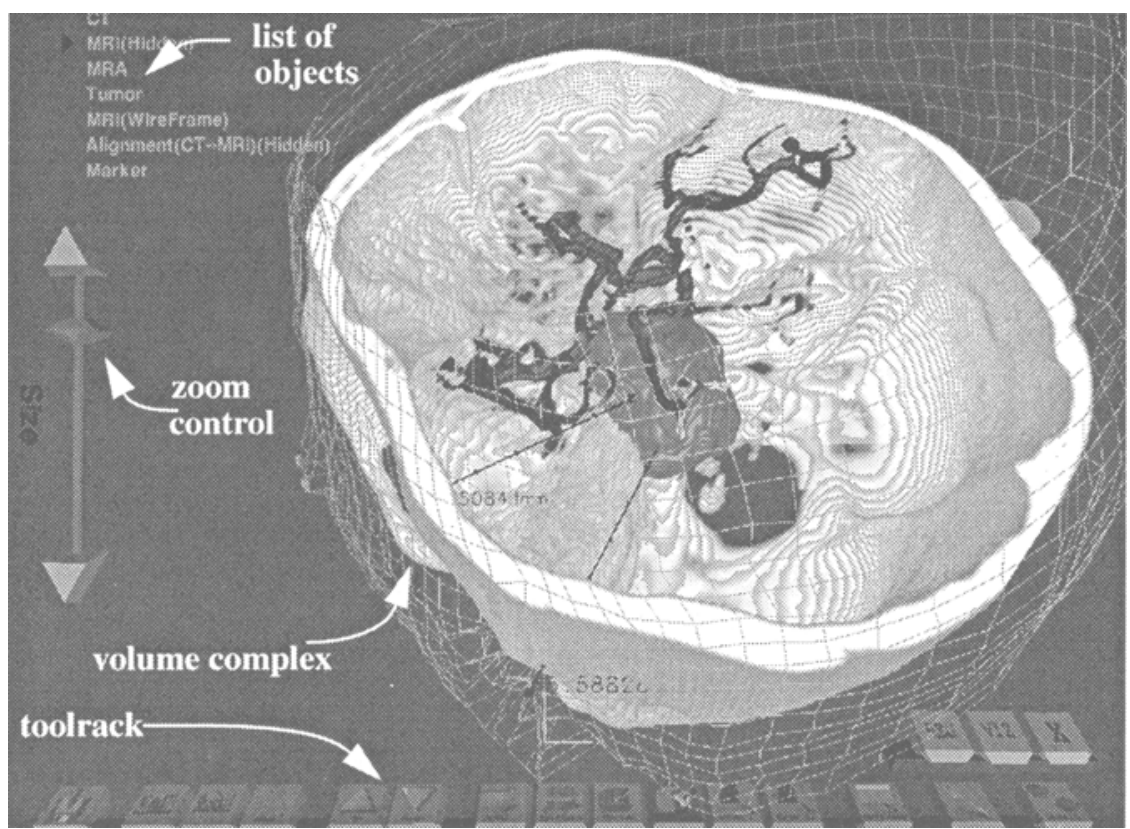

Fig. 1. The multimodal volume: CT, MRA and MRI fused. Markers show the approach

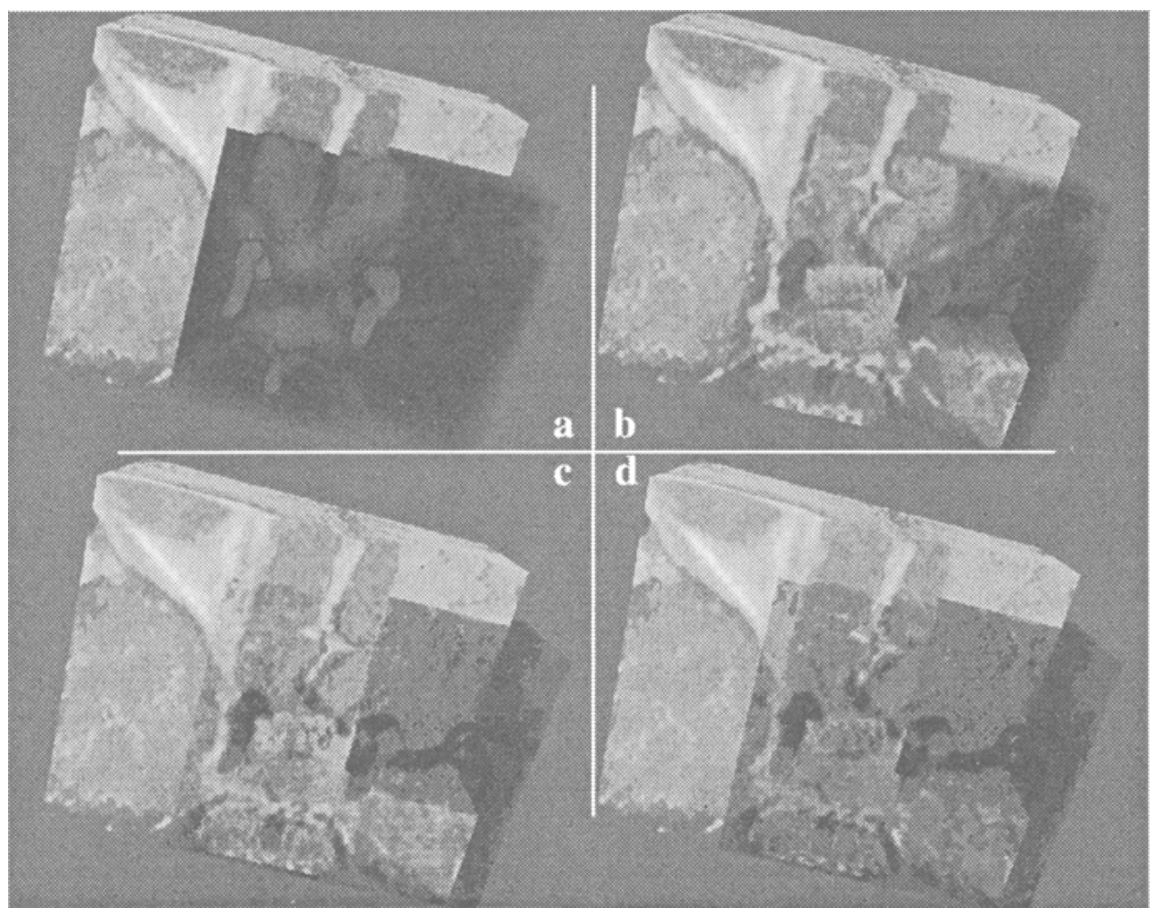

Fig. 2. Multimodal volumes rendered using 3D textures: (a) MRA without mask; (b) MRA with mask; (c, d) using the 'multisampling mask' approach, (c) MRI rendered first, (d) MRA first 

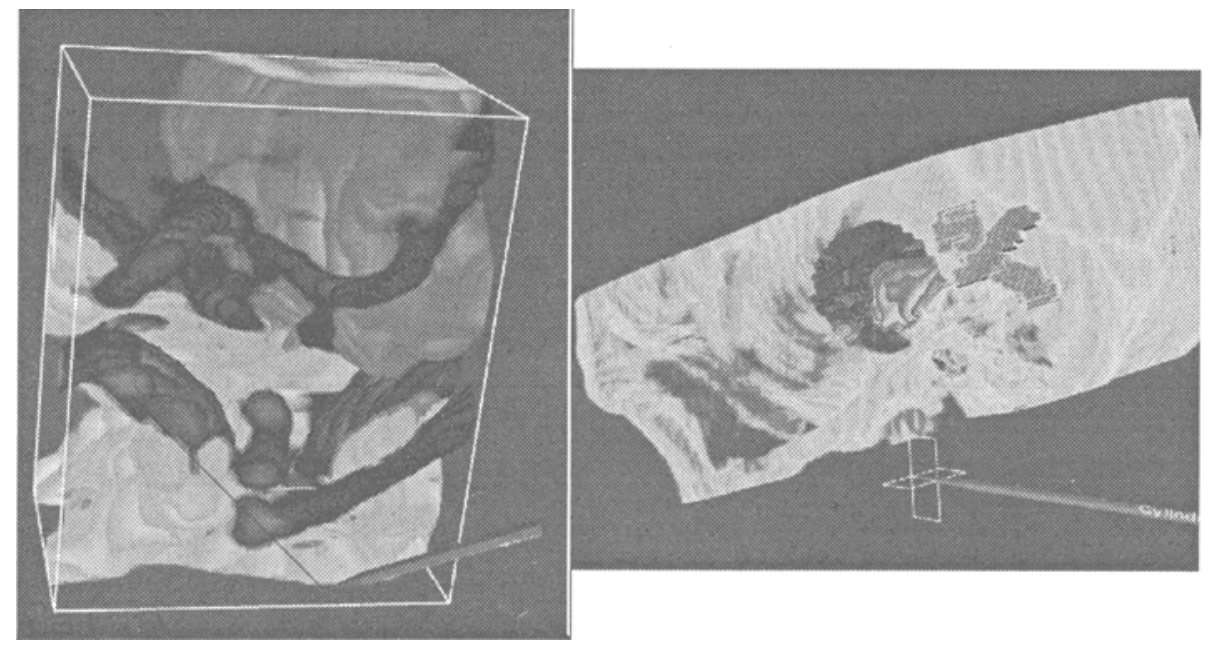

Fig. 3. Volume tools: (a) Roaming around objects; (b) Voxel editor: eraser and paint (dark cross)
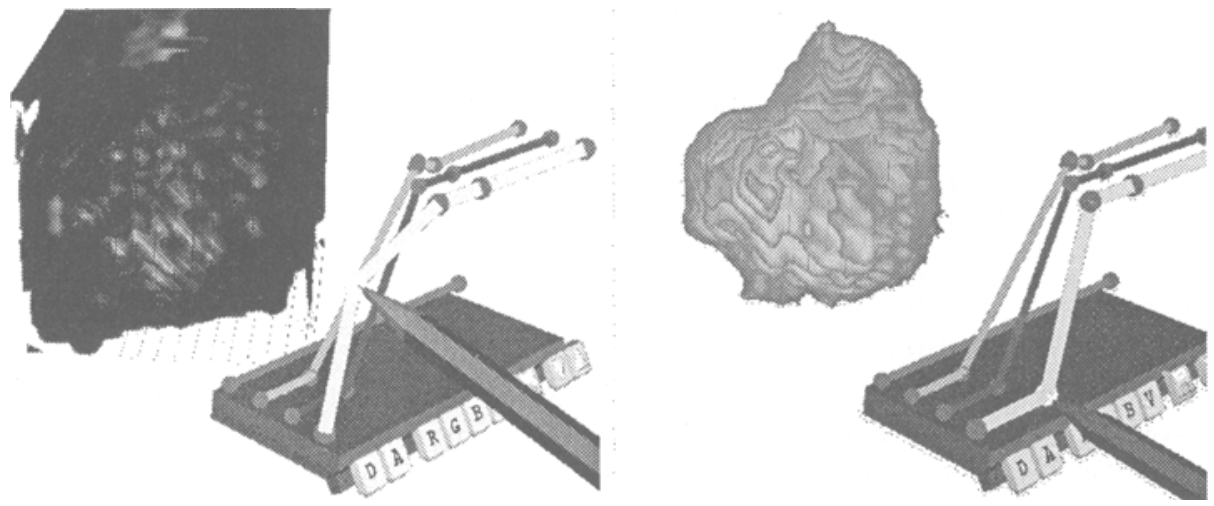

Fig. 4. Tumor highlighting using CLUT control: before and after transparency is adjusted

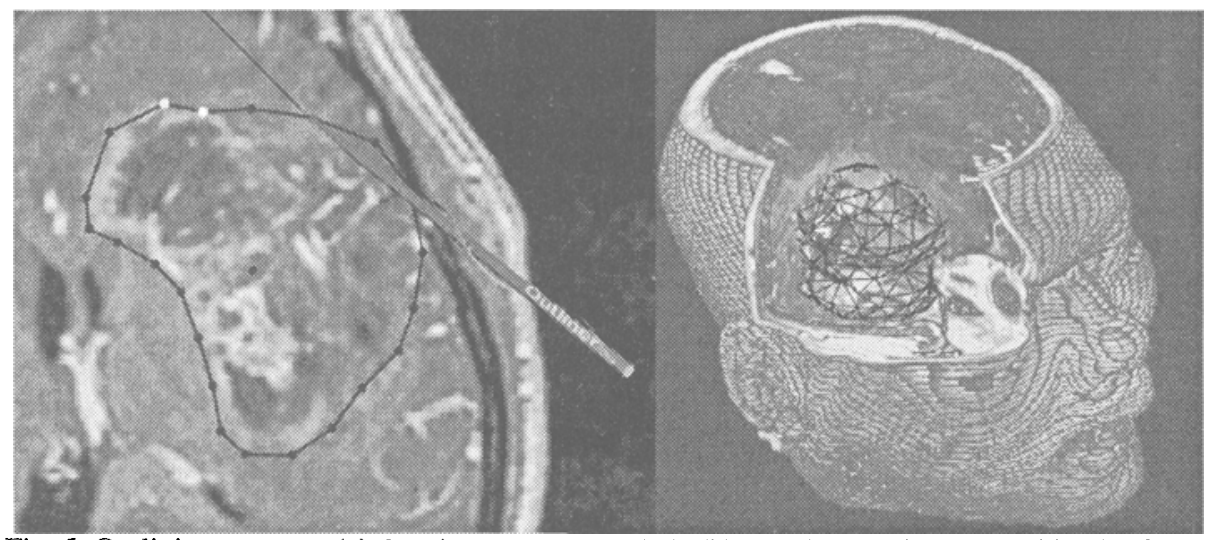

Fig. 5. Outlining a tumor: (a) drawing contours (axial); (b) building mesh and checking its fit 